\title{
Enhanced laser intensity and ion acceleration due to self-focusing in relativistically transparent ultrathin targets
}

\author{
T. P. Frazer $\odot,{ }^{1}$ R. Wilson $\odot,{ }^{1}$ M. King, ${ }^{1}$ N. M. H. Butler, ${ }^{1}$ D. C. Carroll, ${ }^{2}$ M. J. Duff, ${ }^{1}$ A. Higginson $\odot,{ }^{1}$ J. Jarrett, ${ }^{1}$ \\ Z. E. Davidson $\odot,{ }^{1}$ C. Armstrong, ${ }^{1,2}$ H. Liu, ${ }^{3,2}$ D. Neely, ${ }^{2,1}$ R. J. Gray, ${ }^{1}$ and P. McKenna ${ }^{1,}, *$ \\ ${ }^{1}$ SUPA Department of Physics, University of Strathclyde, Glasgow G4 ONG, United Kingdom \\ ${ }^{2}$ Central Laser Facility, STFC Rutherford Appleton Laboratory, Oxfordshire OX11 OQX, United Kingdom \\ ${ }^{3}$ Beijing National Laboratory for Condensed Matter Physics, Institute of Physics, Chinese Academy of Sciences, Beijing 100190, China
}

(Received 23 May 2020; accepted 7 September 2020; published 19 October 2020)

\begin{abstract}
Laser-driven proton acceleration from ultrathin foils is investigated experimentally using $f / 3$ and $f / 1$ focusing. Higher energies achieved with $f / 3$ are shown via simulations to result from self-focusing of the laser light in expanding foils that become relativistically transparent, enhancing the intensity. The increase in proton energy is maximized for an optimum initial target thickness, and thus expansion profile, with no enhancement occurring for targets that remain opaque, or with $f / 1$ focusing to close to the laser wavelength. The effect is shown to depend on the drive laser pulse duration.
\end{abstract}

DOI: 10.1103/PhysRevResearch.2.042015

Following the advent of chirped pulse amplification [1], the interaction of relativistically intense laser light ( $>10^{18} \mathrm{~W} \mathrm{~cm}^{-2}$ for a wavelength of $\sim 1 \mu \mathrm{m}$ ) with thin foil targets has been investigated extensively, as a driver for energetic ions [2,3], bright $x$ rays $[4,5]$, and high harmonics [6,7]. Significant effort is focused on ion acceleration due to the potential for application in areas such as oncology $[8,9]$ and fusion [10].

The most widely investigated mechanism for accelerating ions is target normal sheath acceleration (TNSA), in which an electrostatic field of $\mathrm{TV} \mathrm{m}^{-1}$ scale is formed at the rear of a foil target by energetic electrons accelerated from the laserirradiated front side [11]. If the target expands to the extent that it becomes relativistically transparent to the laser light [12-15], additional electron heating occurs over the expanded plasma, enhancing the field and ion acceleration, in what is termed the break-out afterburner (BOA) scheme [16,17]. For targets that remain opaque, the laser radiation pressure drives charge separation fields, resulting in so-called radiation pressure acceleration (RPA) $[18,19]$. RPA is predicted to produce ion beams with favorable spectral, divergence, and intensityscaling properties $[20,21]$. Hybrid schemes involving two or more mechanisms have also been explored. Due to the laser temporal-intensity profile, linearly polarized light of sufficiently high peak intensity can drive both TNSA and RPA over the course of the interaction, producing a moving dual-peaked electric field [22]. The onset of relativistic transparency can further boost the dual-peaked field [23]. This occurs due to additional heating [17] or the direct acceleration of electrons

\footnotetext{
*paul.mckenna@strath.ac.uk
}

Published by the American Physical Society under the terms of the Creative Commons Attribution 4.0 International license. Further distribution of this work must maintain attribution to the author(s) and the published article's title, journal citation, and DOI. to form a high-energy jet, as the remainder of the laser pulse propagates through the expanding target [24-26]. Previously, we demonstrated that this hybrid scheme can accelerate protons to near $100 \mathrm{MeV}$ [23].

The maximum energy to which ions are accelerated scales with the laser intensity, with a positive power-law exponent that varies with the acceleration mechanism and laser pulse parameters [3]. For fixed laser energy and pulse duration, maximizing the intensity by focusing to a near-wavelengthsized focal spot does not, however, necessarily result in higher-energy ions. Recent studies, involving relatively thick foils, have shown that self-generated magnetic fields [27] and unfavorable changes to the temperature and divergence of the energetic electron population injected into the foil [28] can result in lower-energy TNSA ions compared to that expected from intensity scaling laws.

In this Rapid Communication, we report on an investigation of the influence of laser focusing on proton acceleration in the ultrathin target regime, for which the highest-energy ions have been achieved to date. We compare the interaction physics resulting from the use of $f / 3$ and $f / 1$ focusing geometries-the latter achieved using a focusing plasma optic [29-31]. Although $f / 1$ focusing produces a smaller nominal (in vacuum) laser focal spot size and thus higher nominal peak intensity, more efficient proton acceleration to higher energies is achieved with the $f / 3$ geometry for the case of relativistically transparent expanding ultrathin foils. Particle-in-cell (PIC) simulations reveal that in the $f / 3$ case, self-focusing [32] in the expanding plasma produces a near-diffractionlimited focal spot, resulting in up to an order of magnitude higher focused intensity. We also investigate the extent to which this intensity enhancement is expected in the case of the short-pulse, ultrahigh-intensity regime that will soon be accessible using multi-petawatt lasers.

The experiment was performed using the Vulcan petawatt laser at the Rutherford Appleton Laboratory, U.K., to deliver pulses of $p$-polarized, 1054-nm wavelength $\left(\lambda_{L}\right)$ laser light, 

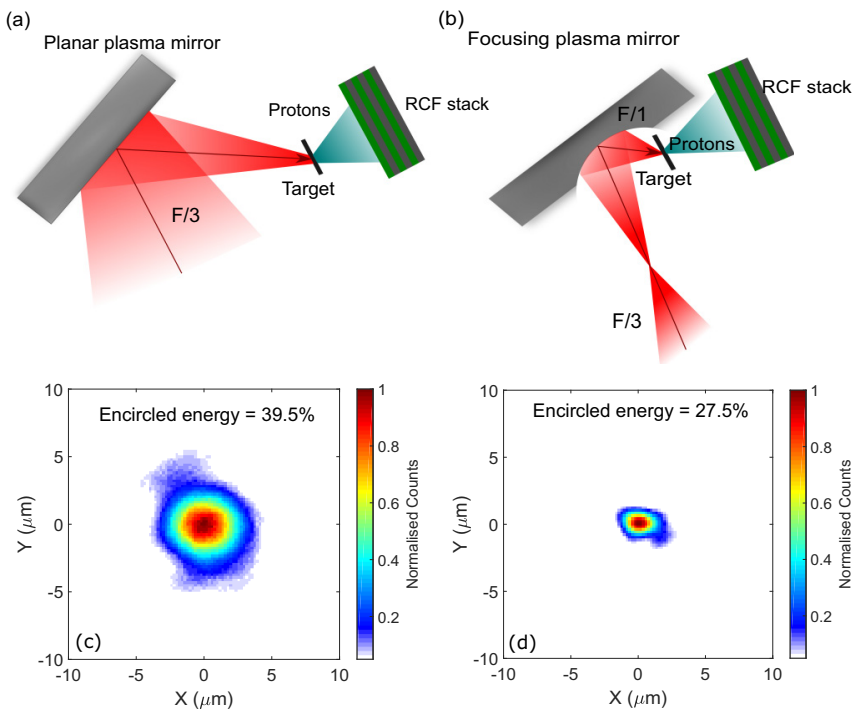

FIG. 1. Schematic of the plasma mirror configurations used for (a) $f / 3$ and (b) $f / 1$ focusing, employing planar and ellipsoidal geometries, respectively. The spatially and energy-resolved dose distribution of the beam of protons accelerated from the ultrathin target foil was characterized using stacked radiochromic film (RCF). Example measured focal spot spatial-energy distributions (at low power) for (c) $f / 3$ and (d) $f / 1$ focusing. The measured encircled energy within the FWHM of each spot is stated.

with duration $\left(\tau_{L}\right)$ equal to $(0.9 \pm 0.1)$ ps and energy equal to $(220 \pm 50) \mathrm{J}$ (on target). The light was focused using a $f / 3$ off-axis parabolic mirror, off a planar plasma mirror (PPM) as illustrated in Fig. 1(a), to a nominal spot size of $\phi_{L}=(4.5 \pm$ $0.9) \mu \mathrm{m}$ [full width at half maximum (FWHM)]. The nominal (calculated) on-target intensity $I_{L}$ was $(3 \pm 2) \times 10^{20} \mathrm{~W} \mathrm{~cm}^{-2}$ over the range of shots. Note that these conditions are very similar to those of our prior work (using the same laser and targets) reported in Ref. [23].

In the second configuration, the PPM was replaced by an ellipsoidal focusing plasma mirror (FPM) to achieve $f / 1$ focusing, as shown in Fig. 1(b). This optic reimages the $f / 3$ focal spot with a demagnification of $\times 3$, reducing $\phi_{L}$ to $1.5 \mu \mathrm{m}$ (FWHM) and increasing the nominal intensity by up to $\times 9$, for the same plasma reflectivity $[30,33]$. Compared to the $f / 3$ configuration, the encircled energy within focal spot FWHM was reduced by between $25 \%$ and $30 \%$. The encircled energy was determined by measuring the laser focal spot distribution at low laser power (to prevent damaging the objective optic and CCD camera-alternative schemes for removing energy and reimaging the focal spot at full power were not feasible due to the rapid beam expansion with the $f / 1$ geometry). Example measurements are shown in Figs. 1(c) and $1(\mathrm{~d})$ for the $f / 3$ and $f / 1$ geometries, respectively. The encircled energy value as measured at low power for each PPM and FPM was corrected to account for any beam wavefront irregularity measured on the corresponding full power shot on the same plasma mirror. The reduction in encircled energy for the $f / 1$ case arises due to the increased sensitivity to small shot-to-shot variations in the laser pointing, which change the energy balance between the focal spot and the lower-intensity wings [30,33]. Factoring this in, together with
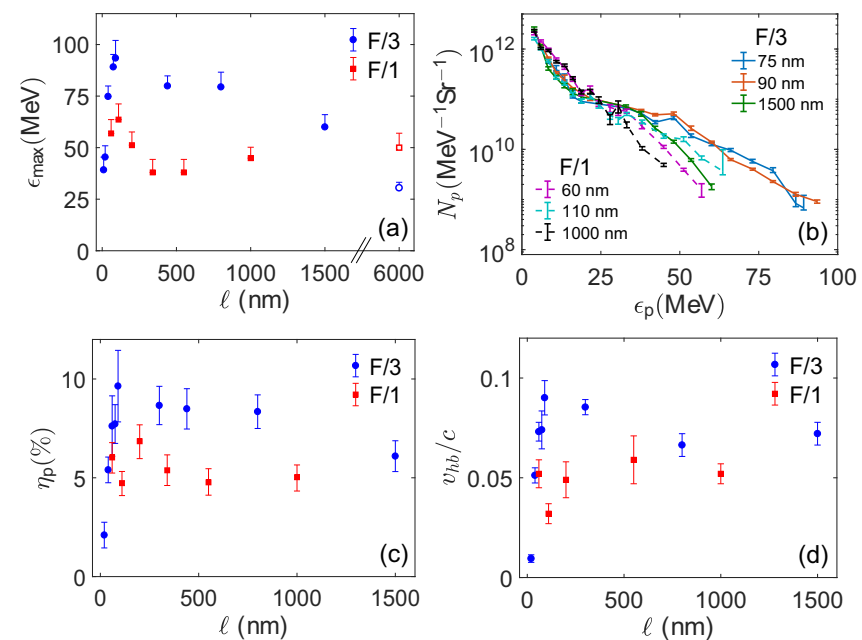

FIG. 2. Experimental results. (a) Maximum proton energy $\left(\epsilon_{\max }\right)$ as a function of target thickness $\ell$. Open symbols correspond to 6 - $\mu$ m-thick Al reference targets. (b) Representative proton energy spectra for given $\ell$, for $f / 1$ and $f / 3$ focusing. (c) Laser-to-proton energy conversion efficiency $\left(\eta_{p}\right)$ and (d) hole-boring velocity $\left(v_{\mathrm{hb}}\right)$ normalized to the speed of light in vacuum $c$, as a function of $\ell$.

the measured laser pulse parameters, the calculated intensity using the FPM was $(1.5 \pm 0.8) \times 10^{21} \mathrm{~W} \mathrm{~cm}^{-2}$. The measured temporal-intensity contrast ratio of the Vulcan pulses was $10^{-10}$ at $500 \mathrm{ps}$ and $10^{-5}$ at $10 \mathrm{ps}$ prior to the peak of the pulse. Both the PPM and FPM were manufactured from the same material and had the same antireflection coating, and both enhance the contrast by approximately two orders of magnitude.

The targets were plastic foils, with thickness $\ell$ varied in the range $60 \mathrm{~nm}-1.5 \mu \mathrm{m}$, irradiated at $(27 \pm 3)^{\circ}$ with respect to the target normal (same as in Ref. [23]). The targets were positioned to a calibrated accuracy of $1.6 \mu \mathrm{m}$ in the depth of focus, using a magnification microscope imaging system with an alignment reference laser of the same central wavelength as the main laser. This degree of alignment accuracy results in an uncertainty of $\pm 10 \%$ in the on-target peak laser intensity. The spatial and spectral dose distributions of the proton beam were measured using stacked radiochromic film (RCF) [34], interspersed with Mylar, iron, and copper filters. The stack was positioned $45 \mathrm{~mm}$ from the target and orientated such that protons accelerated along both the target normal and laser axes were measured. The spectrum of light back-reflected from the target (comprising harmonic light generated and the laser light) was also measured.

To check for consistency with previous results on proton acceleration using the FPM setup, the beam of protons produced from $6-\mu \mathrm{m}$-thick $\mathrm{Al}$ reference targets, for which TNSA is the dominant acceleration mechanism and the target remains opaque, was characterized. Maximum proton energies of $\sim 50 \mathrm{MeV}$ were measured with $f / 1$, compared to $\sim 30 \mathrm{MeV}$ for the $f / 3$ case, as shown in Fig. 2(a). The higher proton energy results from the intensity enhancement due to tighter focusing, and is in good agreement with results previously reported by our group for the same target and FPM focusing setup [30]. 
Having completed these reference measurements, a scan of proton beam measurements as a function of thickness was performed for the plastic targets. Figure 2(b) presents example measured proton energy spectra, for stated values of $\ell$, for both $f / 1$ and $f / 3$ focusing. The vertical error bars are defined by the uncertainty in the proton dose $N_{p}$ based on the RCF calibration. The $f / 3$ data are the same as presented in Ref. [23]. The maximum proton energy $\epsilon_{\max }$ and laser-to-proton energy conversion efficiency $\eta_{p}$ are shown as a function of $\ell$ in Figs. 2(a) and 2(c), respectively. Although the nominal intensity (without self-focusing) is higher in the $f / 1$ case, with $\phi_{L}=1.5 \mu \mathrm{m}$, both $\epsilon_{\max }$ and $\eta_{p}$ are higher for $f / 3$ focusing. The highest value of $\epsilon_{\max }$ was in the range 94-101 $\mathrm{MeV}$, measured for $\ell \sim 100 \mathrm{~nm}$ and $f / 3$ focusing. The highest values of $\epsilon_{\max }$ measured for the $f / 1$ case were in the range 64-72 MeV, also for $\ell \sim 100 \mathrm{~nm}$. A similar factor reduction $(\sim 40 \%)$ in $\eta_{p}$ is observed compared to the $f / 3$ values.

The maximum velocity at which the laser bores into the plasma (the laser hole-boring velocity $v_{\mathrm{hb}}$ ) was also determined, by measuring the relativistic Doppler shift in the wavelength of the second harmonic light generated at the critical density surface moving under the effect of the laser radiation pressure [35]. The results are presented in Fig. 2(d), where $v_{\mathrm{hb}}$ is calculated from the maximum wavelength where the signal remains distinguishable above the background level. Note that $v_{\mathrm{hb}}$ reduces rapidly in ultrathin foils as transparency occurs earlier on the rising edge of the laser intensity profile. $v_{\mathrm{hb}}$ is generally higher for the $f / 3$ case and in particular for the $\ell=50-200 \mathrm{~nm}$ range for which $\eta_{p}$ and $\epsilon_{\max }$ are maximized. As $v_{\mathrm{hb}}$ scales with laser intensity [36], this points to a higher laser intensity being achieved in the case of $f / 3$, in spite of the nominally larger focal spot size and comparable laser energy and $\tau_{L}$.

This counterintuitive behavior was investigated using the fully relativistic PIC code EPOCH [37] in two and three dimensions (2D and 3D). The 2D box was defined to be $130 \mu \mathrm{m} \times$ $70 \mu \mathrm{m}$, with $26000 \times 5760$ simulation cells, while in $3 \mathrm{D}$ the box was set as $20 \mu \mathrm{m} \times 10 \mu \mathrm{m} \times 10 \mu \mathrm{m}$, with $1000 \times$ $720 \times 720$ simulation cells. The boundaries in both cases were set as free space and the targets were initialized as a uniform mixture of $\mathrm{C}^{6+}$ and $\mathrm{H}^{+}$ions (representative of plastic foils). In the $2 \mathrm{D}$ case, the initial electron density was equal to $420 n_{c}$, where $n_{c}$ is the critical density for the fundamental laser frequency, i.e., the density below which the target becomes transparent to the laser pulse, and the initial temperature was $10 \mathrm{keV}$. The targets in the $3 \mathrm{D}$ case had an equivalent areal density to the 2D 50-nm target, expanded to a Gaussian profile along the laser axis with a FWHM equal to $0.5 \mu \mathrm{m}$, for all focusing conditions, to reduce the spatial resolution required and thereby make the computation feasible. This is justified because experimentally the target electron population expands very early in the interaction due to the finite temporal-intensity contrast of the laser pulse [38,39]. The laser pulse, defined as a Gaussian profile both spatially and temporally, was focused and incident at an angle of $30^{\circ}$ to the target normal axis in both the $2 \mathrm{D}$ and $3 \mathrm{D}$ cases, to closely match the experiment. 2D simulations were performed for $\tau_{L}=400 \mathrm{fs}$ and $\tau_{L}=25 \mathrm{fs}$. Here, $400 \mathrm{fs}$ is shorter than in the experiment and is justified due to the exaggerated ion expansion in a $2 \mathrm{D}$ simulation as a result of the reduced degrees
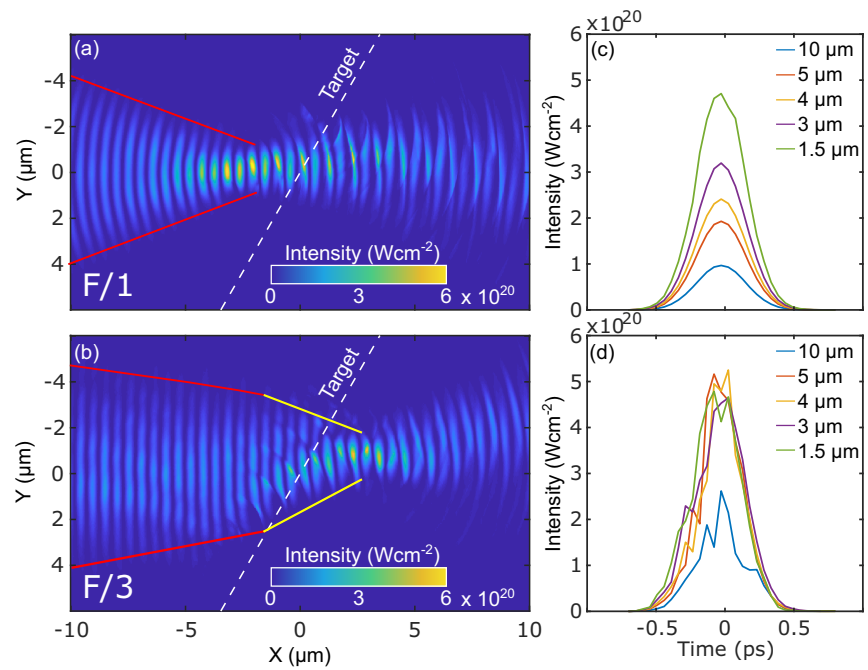

FIG. 3. 2D PIC simulation results. (a) Spatial map of the instantaneous laser intensity for $f / 1$ focusing onto a $\ell=75 \mathrm{~nm} \mathrm{CH}$ target. The red lines highlight the focusing cone of the incident laser light. (b) Same, for the $f / 3$ focusing case, with the yellow lines highlighting the cone of the additional self-focusing. (c) Temporal evolution of the peak focused 2D laser intensity, for given $\phi_{L}$ between 1.5 and $10 \mu \mathrm{m}$, propagating in vacuum. (d) Same, for the interaction with a $\ell=75 \mathrm{~nm} \mathrm{CH}$ target expanded to the point at which the target becomes relativistically transparent to the laser light. The peak $2 \mathrm{D}$ intensity reaches a similar magnitude for all $\phi_{L}$ in the range $1.5-5 \mu \mathrm{m}$ due to self-focusing. $t=0 \mathrm{ps}$ is defined as the time at which the peak of the incident laser pulse interacts with the target.

of freedom [23]. It is also important to note here that the laser intensity scales linearly with focal spot size due to the 2D nature of the simulations, having only one longitudinal and one transverse dimension. The 3D simulations were limited to the 25-fs case (due to computational restrictions), with the energy fixed to give an intensity of $2.5 \times 10^{22} \mathrm{~W} \mathrm{~cm} \mathrm{~cm}^{-2}$ for a spot size of $1.2 \mu \mathrm{m}$. In separate parameter scans, $\ell$ was varied in the range $20 \mathrm{~nm}-1 \mu \mathrm{m}$ and $\phi_{L}$ was varied from 1.5 to $10 \mu \mathrm{m}$, for otherwise fixed parameters. The $2 \mathrm{D}$ simulations were conducted for very similar parameters to those employed in our prior work reported in Ref. [23], except that in the present case the focal spot size, and therefore on-target intensity, is varied.

Figures 3(a) and 3(b) show 2D intensity maps of the laser pulse with an input focal spot size of $1.5 \mu \mathrm{m}(f / 1)$ and $5 \mu \mathrm{m}$ $(f / 3)$, respectively, at a fixed time in the interaction with a $\ell=75 \mathrm{~nm} \mathrm{CH}$ target. The initial target position is marked by the dashed white line and the nominal focusing cone and selffocusing cone are shown as red and yellow lines, respectively. In Fig. 3(a), the laser pulse focuses to an initial spot of $1.5 \mu \mathrm{m}$, with little scope for additional, plasma-induced, focusing $\left(\phi_{L}\right.$ being close to the diffraction limit of $\sim 1.3 \mu \mathrm{m})$. By contrast, in Fig. 3(b) the laser light is focused initially to $\phi_{L}=5 \mu \mathrm{m}$ at the target surface and then undergoes additional self-focusing within the expanding plasma, as it becomes transparent. The final focal spot size, and instantaneous intensity, is comparable to the $\phi_{L}=1.5 \mu \mathrm{m}$ case.

The role of plasma-induced self-focusing was verified by comparing the peak intensities achieved when propagating laser pulses in vacuum and through a plasma produced by an 

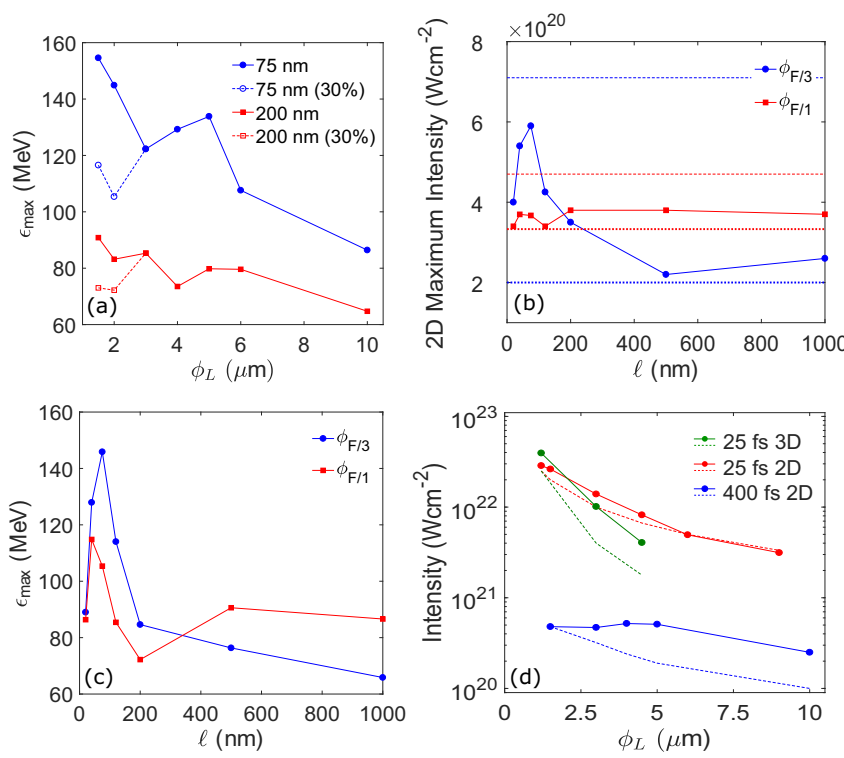

FIG. 4. PIC simulation results. (a) 2D PIC: $\epsilon_{\max }$ scaling with $\phi_{L}$ for $\ell=75 \mathrm{~nm}$ and $\ell=200 \mathrm{~nm}$. Open symbols are for a $30 \%$ reduction in encircled energy. (b) 2D PIC: Maximum intensity achieved as a function of $\ell$ for the nominal $\phi_{L}=1.5 \mu \mathrm{m}$ and $\phi_{L}=5 \mu \mathrm{m}$ cases. Dotted lines indicate the peak nominal intensity and the dashed lines represent the maximum intensity achievable, corresponding to focusing to the diffraction limit. (c) 2D PIC: $\epsilon_{\max }$ as a function of $\ell$ for both focusing cases. (d) 2D and 3D PIC: Peak intensity as a function of $\phi_{L}$ for $\tau_{L}=400 \mathrm{fs}$ (blue) and $\tau_{L}=25$ fs (red) in 2D simulations. 3D PIC results for $\tau_{L}=25 \mathrm{fs}$ are shown in green. The target is an expanding $\ell=100 \mathrm{~nm}$ foil in all cases. Dashed lines represent the nominal (vacuum) maximum intensity. The intensities of the 2D cases are scaled by the square of the enhancement over the nominal (vacuum) intensity, to enable direct comparison with the $3 \mathrm{D}$ results.

expanding $\ell=75 \mathrm{~nm} \mathrm{CH}$ target, for $\phi_{L}$ ranging from 1.5 to $10 \mu \mathrm{m}$, as shown in Fig. 3(c) (vacuum) and Fig. 3(d) (plasma). With the exception of $\phi_{L}=10 \mu \mathrm{m}$, a similar peak intensity is achieved for all $\phi_{L}$ due to self-focusing to near the diffraction limit. The final intensity for the $\phi_{L}=10 \mu \mathrm{m}$ case is also higher than in vacuum, but $\phi_{L}$ is too large compared to the propagation length in the plasma to achieve self-focusing to a value close to $\lambda_{L}$. Moreover, because the nominal intensity (before self-focusing) is reduced, the onset of transparency is delayed, further reducing the influence of self-focusing. Thus an upper limit for $\phi_{L}$ exists below which self-focusing can drive the pulses to comparable intensities by achieving a near-diffraction-limited focal spot.

Figure 4(a) shows how $\epsilon_{\max }$ varies with $\phi_{L}$ in the simulation results for two example target thicknesses, $\ell=75 \mathrm{~nm}$ and $\ell=200 \mathrm{~nm}$. Note that due to the reduced dimensionality of the simulations, the absolute values for $\epsilon_{\max }$ are higher than measured experimentally [23]. Additional simulation results for a reduction of $30 \%$ in laser energy are included for $\phi_{L}=1.5 \mu \mathrm{m}$ and $\phi_{L}=2 \mu \mathrm{m}$. This reduction in energy in the simulations is to correctly take account of the reduction in encircled energy arising from the potential alignment errors when using the FPM in the experiment, as discussed above. For $\ell=200 \mathrm{~nm}, \epsilon_{\max }$ increases relatively slowly with decreasing $\phi_{L}$, and is highest for the smallest spot size. The target becomes transparent very late in the interaction and thus self-focusing is not evident. By contrast, the $\ell=75 \mathrm{~nm}$ target becomes relativistically transparent near the peak of the interaction, enabling self-focusing and a resulting enhancement in $\epsilon_{\max }$ for $3<\phi_{L}<6 \mu \mathrm{m}$, beyond the overall trend of $\epsilon_{\max }$ increasing with decreasing $\phi_{L}$. Importantly, although the highest $\epsilon_{\max }$ is still obtained for the smallest $\phi_{L}$ when the laser energy is constant, this is not the case when factoring in a reduction of $30 \%$ in encircled energy for the tightest focus. The self-focusing induced for $\phi_{L}=5 \mu \mathrm{m}$ produces the highest intensity and thus $\epsilon_{\max }$.

By investigating the specific cases of $\phi_{L}=5 \mu \mathrm{m}$ and $\phi_{L}=1.5 \mu \mathrm{m}$, the differences in the interaction physics for the $f / 3$ and $f / 1$ focusing in the experiment were explored as a function of $\ell$. The peak intensity in the $\phi_{L}=1.5 \mu \mathrm{m}$ case is lowered by $30 \%$ to account for the reduction in encircled energy with the $f / 1$ optic. Note that the nominal (vacuum) intensity remains higher than that of the $f / 3$ case. The results are shown in Fig. 4(b). Compared to the nominal focus case, it can be seen that the $\phi_{L}=1.5 \mu \mathrm{m}$ simulations exhibit minimal enhancement across the full range of $\ell$. The $\phi_{L}=5 \mu \mathrm{m}$ results show minimal intensity enhancement for $\ell \geqslant 500 \mathrm{~nm}$, due to the target remaining opaque and the laser undergoing minimal self-focusing. By contrast, for thinner targets that expand to the point of relativistic transparency, the laser pulse self-focuses as it propagates within the target to produce significant intensity enhancement, approaching the maximum achievable intensity at the diffraction limit for $\ell=100 \mathrm{~nm}$, for which transparency occurs near the peak of the interaction.

Figure 4(c) shows how $\epsilon_{\max }$ varies as a function of $\ell$ for both focusing cases. Importantly, consistent with the experimental results, compared to $f / 3$, the $f / 1\left(\phi_{L}=1.5 \mu \mathrm{m}\right)$ case results in smaller $\epsilon_{\max }$ for the thinner targets $(\ell<400 \mathrm{~nm})$. $\epsilon_{\max }$ is higher for $f / 1$ focusing compared to $f / 3$ for relatively thick targets $(\ell>400 \mathrm{~nm})$, for which there is limited selffocusing occurring and thus limited intensity enhancement. As shown in Fig. 2(a) and in Ref. [30], our experimental results for thick foils $(\ell=6 \mu \mathrm{m})$ exhibit higher $\epsilon_{\max }$ with $f / 1$ compared to $f / 3(50 \mathrm{MeV}$ compared to $30 \mathrm{MeV})$, as expected due to the absence of self-focusing in opaque targets. For the thickness range up to $1.5 \mu \mathrm{m}$, as explored in Fig. 2, we do not observe a higher $\epsilon_{\max }$ for $f / 1$, though the measured values of $\epsilon_{\max }, \eta_{p}$, and $v_{\mathrm{hb}}$ for both focusing conditions all tend to converge with increasing thickness.

Finally, we consider whether plasma self-focusing and intensity enhancement occurs in the case of shorter and higher-intensity pulses available at a number of upcoming multi-petawatt laser facilities, as this could potentially be used to further increase the intensity frontier. Figure 4(d) compares 2D PIC results for peak intensity scaling as a function of $\phi_{L}$ for $\left[\tau_{L}=25 \mathrm{fs} ; \lambda_{L}=0.8 \mu \mathrm{m}\right]$ and $\left[\tau_{L}=400 \mathrm{fs}\right.$; $\left.\lambda_{L}=1 \mu \mathrm{m}\right]$. Here, $\ell=100 \mathrm{~nm}$ for both cases. For the $\tau_{L}=$ 400 fs case discussed above, intensity enhancement over the nominal vacuum intensity is observed for all $\phi_{L}$ (except the smallest spot), with an optimum $\phi_{L} \sim 4 \mu \mathrm{m}$. In contrast, minimal enhancement is achieved for all $\phi_{L}$ for the $\tau_{L}=25$ fs case in $2 \mathrm{D}$, indicating that self-focusing does not play a significant role. 3D simulations are feasible in the case of 
the shorter pulse and, as shown in Fig. 4(d), some intensity enhancement over focusing in vacuum is produced at all $\phi_{L}$, including the smallest spot $\left(\phi_{L}=1.2 \mu \mathrm{m}\right)$ because it is not at the diffraction limit (for $\lambda_{L}=800 \mathrm{~nm}$ ). However, in contrast to the longer pulse case, in both the $2 \mathrm{D}$ and $3 \mathrm{D}$ simulations, the highest intensity is achieved for the smallest nominal $\phi_{L}$ (i.e., initial tight focusing), indicating that self-focusing does not play a significant role because the plasma does not expand to the same degree over the duration of the shorter pulse. Pre-expanding the target via the use of a controlled prepulse may induce plasma self-focusing to enhance intensity and this will be investigated in a future study.

It was shown previously that laser self-focusing and filamentation in density gradients at the front side of thick foils influence the properties of beams of TNSA ions [40-42] and ion energy enhancement due to laser self-focusing in a layer of near-critical density plasma at the front side of an opaque foil has also been demonstrated [43]. The present results reveal that self-focusing also plays an important role in ion acceleration from ultrathin foils that become relativistically transparent during the interaction. Thus the nominal spot size to which the laser pulse is focused is important for the optimization of acceleration mechanisms employing expanding ultrathin foil targets, such as the BOA, RPA, and hybrid TNSA-RPA schemes. Selffocusing is likely to have also occurred in our study reported in Ref. [23], performed under similar $f / 3$ focusing conditions. That study focused on investigating the acceleration mechanism as a function of target thickness and did not explore the role of focal spot size. Our present results further reveal that for the case of ultrahigh-intensity contrast, short, multi-petawatt laser pulses, limited self-focusing occurs due to the limited extent of plasma expansion during the interaction.

Data associated with research published in this Rapid Communication can be freely accessed [44].

We acknowledge the Central Laser Facility staff, financial support by EPSRC (EP/R006202/1, EP/M018091/1, and $\mathrm{EP} / \mathrm{K} 022415 / 1$ ), use of the ARCHER high performance computer (via Plasma Physics HEC Consortium Grant No. EP/R029148/1), and the University of Cambridge Research Computing Service [45] (funded by Grant No. $\mathrm{EP} / \mathrm{P020259/1).} \mathrm{EPOCH}$ was developed under EPSRC Grant No. EP/G054940/1.
[1] D. Strickland and G. Mourou, Opt. Commun. 56, 219 (1985).

[2] H. Daido, M. Nishiuchi, and A. S. Pirozhkov, Rep. Prog. Phys. 75, 056401 (2012).

[3] A. Macchi, M. Borghesi, and M. Passoni, Rev. Mod. Phys. 85, 751 (2013)

[4] J. Colgan et al., Phys. Rev. Lett. 110, 125001 (2013).

[5] P. Norreys et al., Phys. Plasmas 6, 2150 (1999).

[6] B. Dromey et al., Nat. Phys. 2, 456 (2006).

[7] B. Dromey et al., Phys. Rev. Lett. 99, 085001 (2007).

[8] S. V. Bulanov and V. S. Khoroshkov, Plasma Phys. Rep. 28, 453 (2002).

[9] K. W. D. Ledingham, P. R. Bolton, N. Shikazono, and C.-M. C. Ma, Appl. Sci. 4, 402 (2014).

[10] M. Roth et al., Phys. Rev. Lett. 86, 436 (2001).

[11] S. C. Wilks, A. B. Langdon, T. E. Cowan, M. Roth, M. Singh, S. Hatchett, M. H. Key, D. Pennington, A. MacKinnon, and R. A. Snavely, Phys. Plasmas 8, 542 (2001).

[12] V. A. Vshivkov, N. M. Naumova, F. Pegoraro, and S. V. Bulanov, Phys. Plasmas 5, 2727 (1998).

[13] V. I. Eremin, A. V. Korzhimanov, and A. V. Kim, Phys. Plasmas 17, 043102 (2010).

[14] B. Gonzalez-Izquierdo et al., Nat. Phys. 12, 505 (2016).

[15] B. Gonzalez-Izquierdo et al., Nat. Commun. 7, 12891 (2016).

[16] L. Yin, B. J. Albright, B. M. Hegelich, and J. C. Fernández, Laser Part. Beams 24, 291 (2006).

[17] B. M. Hegelich et al., New J. Phys. 15, 085015 (2013).

[18] T. Esirkepov, M. Borghesi, S. V. Bulanov, G. Mourou, and T. Tajima, Phys. Rev. Lett. 92, 175003 (2004).

[19] A. P. L. Robinson, M. Zepf, S. Kar, R. G. Evans, and C. Bellei, New J. Phys. 10, 013021 (2008).

[20] S. Kar et al., Phys. Rev. Lett. 109, 185006 (2012).

[21] I. J. Kim et al., Phys. Plasmas 23, 070701 (2016).

[22] B. Qiao, S. Kar, M. Geissler, P. Gibbon, M. Zepf, and M. Borghesi, Phys. Rev. Lett. 108, 115002 (2012).
[23] A. Higginson et al., Nat. Commun. 9, 724 (2018).

[24] H. W. Powell et al., New J. Phys. 17, 103033 (2015).

[25] S. Palaniyappan, C. Huang, D. C. Gautier, C. E. Hamilton, M. A. Santiago, C. Kreuzer, A. B. Sefkow, R. C. Shah, and J. C. Fernandez, Nat. Commun. 6, 10170 (2015).

[26] M. King et al., Nucl. Instrum. Methods Phys. Res., Sect. A 829, 163 (2016).

[27] M. Nakatsutsumi et al., Nat. Commun. 9, 280 (2018).

[28] N. Dover et al., Phys. Rev. Lett. 124, 084802 (2020).

[29] M. Nakatsutsumi, A. Kon, S. Buffechoux, P. Audebert, J. Fuchs, and R. Kodama, Opt. Lett. 35, 2314 (2010).

[30] R. Wilson et al., Phys. Plasmas 23, 033106 (2016).

[31] D. Kumar et al., Matter Radiat. Extremes 4, 024402 (2019).

[32] M. R. Siegrist, Opt. Commun. 16, 402 (1976).

[33] R. Wilson et al., Quantum Beam Sci. 2, 1 (2018).

[34] F. Nurnberg et al., Rev. Sci. Instrum. 80, 033301 (2009).

[35] B. Gonzalez-Izquierdo et al., Appl. Sci. 8, 336 (2018).

[36] S. C. Wilks, W. L. Kruer, M. Tabak, and A. B. Langdon, Phys. Rev. Lett. 69, 1383 (1992).

[37] T. D. Arber et al., Plasma Phys. Controlled Fusion 57, 113001 (2015).

[38] M. J. Duff et al., Sci. Rep. 10, 105 (2020).

[39] S. D. R. Williamson et al., New J. Phys. 22, 053044 (2020).

[40] P. McKenna et al., Laser Part. Beams 26, 591 (2008).

[41] R. J. Gray et al., New J. Phys. 16, 113075 (2014).

[42] D. Rusby, R. Gray, N. Butler, R. Dance, G. Scott, V. Bagnoud, B. Zielbauer, P. McKenna, and D. Neely, EPJ Web Conf. 167, 02001 (2018).

[43] J. H. Bin et al., Phys. Rev. Lett. 115, 064801 (2015).

[44] T. Frazer et al., Data for Enhanced laser intensity and ion acceleration due to self-focusing in relativistically transparent ultrathin targets, University of Strathclyde, https://doi.org/10. 15129/70e9031f-b6d9-45bd-af2a-0945aad9f8d6 (2020).

[45] http://www.hpc.cam.ac.uk/. 